\title{
Design and Synthesis of New Fullerene Derivatives for Organic Solar Cells
}

\author{
Jianping Lu ${ }^{1}$, Salima Alem ${ }^{1}$, Afshin Dadvand ${ }^{1}$, Jianfu Ding ${ }^{2}$, and Ye Tao ${ }^{1}$ \\ ${ }^{1}$ Advanced Electronic and Photonic Research Centre \\ ${ }^{2}$ Security and Disruptive Technologies Research Centre \\ National Research Council of Canada, Ottawa, Canada, K1A 0R6 \\ Phone/Fax number:+001 613 9901651, e-mail: Jianping.lu@nrc.ca
}

\begin{abstract}
Bulk heterojunction (BHJ) organic solar cells based on p-type polymers and n-type fullerene derivatives have attracted increasing attention due to their promising potential for providing low-cost solar electricity. In this paper, we will introduce our recent work on the development of new fullerene derivatives. Through $\mathrm{Rh}$ complex catalyzed coupling reactions and cycloaddition reactions, we attached several organic functional groups to the fullerene skeleton. It was found that the organic substituents had little impact on the fullerene energy levels. Instead, the organic substituents have huge impact on the other physical properties of the resulting fullerene derivatives, such as solubility, crystallinity, and electron mobility. We designed and synthesized a novel series of alkoxy substituted indene derivatives as solubilizing groups for fullerenes. Preliminary experimental results demonstrated that these 5alkoxyindene modified $\mathrm{C} 70$ derivatives are superior to widely used PC71BM when blended with poly[N-heptadecanyl-2,7carbazole-alt-5,5-(4',7'-di-2-thienyl-2',1',3'- benzothiadiazole)] (PCDTBT) in BHJ solar cells. The device series resistance decreased from $10 \Omega \mathrm{cm}^{2}$ for the PC71BM based device to $4 \Omega$ $\mathrm{cm}^{2}$ for the 5-methoxyindene-C70 monoadduct based device. As a result, the device fill factor increased from 0.60 to 0.69 , and the overall EQE-calibrated power conversion efficiency was enhanced from $5.6 \%$ to $6.2 \%$.
\end{abstract}

\section{Key words}

Fullerene derivatives, Organic solar cells, 5-alkoxyindene, substituent effects

\section{Introduction}

Bulk heterojunction (BHJ) organic solar cells consisting of conjugated p-type polymers and n-type fullerene derivatives have been intensively investigated in both academia and industry over the past two decades due to their unique technique features, such as low material and production cost, light weight, excellent mechanical flexibility, and mass production by high throughput roll-toroll printing and coating techniques. Significant progress has been made in this field and cells with efficiencies exceeding $10 \%$ have been demonstrated due to the substantial development of new donor materials, the morphology control of the BHJ blends at nanometer scale, and interface engineering. [1-2] In contrast to the intensive study on p-type materials, the design and synthesis of new fullerene derivatives for applications in solar cells as electron acceptors are relatively unexplored with most work focusing on the modification of PC61BM ([6,6]-phenyl $\mathrm{C}_{61}$ butyric acid methyl ester) or PC71BM ([6,6]-phenyl $\mathrm{C}_{71}$ butyric acid methyl ester) derivatives.

Fullerene derivatives have attracted significant attention as promising functional components in nanoelectronic devices due to their high electron affinity and mobility. [3] A number of chemical reactions have been developed to attach organic functional groups to the fullerene skeletons, including cycloaddition, the addition of nucleophiles and free radicals, and more recently Rhcatalyzed arylation with aromatic boronic compounds. [4-5] In this work, we attached several organic functional groups to the fullerene skeleton by different reactions with an intention to modify the optoelectronic properties of fullerene derivatives for better performance. Our study showed that the energy levels of the fullerene derivatives mainly depended on the $\pi$ electron system of the fullerene cage, and the substituents had little impact on the fullerene energy levels. However, the organic substituents have huge impact on the other physical properties of the resulting fullerene derivatives, such as solubility, crystallinity, and electron mobility. We designed and synthesized a series of alkoxy substituted indene derivatives as solubilizing groups for fullerenes. Preliminary experimental results demonstrated that these 5-alkoxyindene modified $\mathrm{C} 70$ derivatives are superior to widely used PC71BM when blended with poly[Nheptadecanyl-2,7-carbazole-alt-5,5-(4',7'-di-2-thienyl2',1',3'- benzothiadiazole)] (PCDTBT) in BHJ solar cells. The device series resistance decreased from $10 \Omega \mathrm{cm}^{2}$ for the PC71BM based device to $4 \Omega \mathrm{cm}^{2}$ for the 5methoxyindene-C70 monoadduct based device. As a result, the device fill factor increased from 0.60 to 0.69 , 
and the overall EQE-calibrated power conversion efficiency was enhanced from $5.6 \%$ to $6.2 \%$. In contrast, unsubstituted indene-C70 monoadduct (IC70MA) has a low solubility in organic solvents. As a result, the PCDTBT/IC70MA blend film has a poor morphology, and the overall power conversion efficiency dropped to $4.0 \%$.

\section{Results and discussion}

\section{A. Rh-catalyzed arylation of fullerenes}

Rh-catalyzed arylation of fullerenes using aromatic boronic compounds opens a new avenue for the versatile functionalization of fullerenes with $\pi$-conjugated compounds. We attached oligofluorenes to the C60 molecule as monodisperse oliogofluorenes possess promising optoelectronic properties and are widely used as electron-transporting blue emitters in light-emitting diodes. In addition, we also designed and synthesized an oligofluorene bridged $\mathrm{C} 60$ dimer with an intention to lower its LUMO energy level to match with some low bandgap polymers with low-lying LUMO levels (below $3.9 \mathrm{eV})$. The synthetic route is shown in Scheme 1. The fullerene derivatives were purified first by silica gel column chromatography and then by HPLC using toluene as the eluent, and identified by proton NMR and MALDITOF mass spectrometry. The UV-vis absorption spectra of the synthesized C60 derivatives 1, 5 and PC61BM in chloroform are shown in Figure 1. PC61BM displays an absorption peak at $328 \mathrm{~nm}$, arising from the $\pi-\pi *$ transition of the delocalized $58 \pi$ electrons. It was found that the interaction in ground state between the C60 buckyball and the oligofluorene bridge was weak. This result is not very surprising, considering the conjugation between the oligofluorene bridge and the $\pi$-electron system of C60 is disrupted by a $\mathrm{sp}^{3}$ hybridized carbon. C60 derivatives 1, 5 and PC61BM have similar optical bandgaps, as calculated from their absorption edges. Moreover, cyclic voltammetry (CV) measurement showed that the synthesized C60 derivatives 1 and 5 have LUMO energy levels quite similar to that of PC61BM, located at $\sim 3.94 \mathrm{eV}$. This means that the energy levels of fullerene derivatives mainly depend on the $\pi$ electron system of the C60 skeleton and the oligofluorene substituents have little effect on the energy levels.

The PV response of the synthesized C60 derivatives 1 and 5 were investigated by blending them with PCDTBT at different weight ratios. PCDTBT was selected as the holetransporting material in this study because this polymer has an excellent stability and demonstrated a power conversion efficiency of $6.0 \%$ when blended with PC71BM. [6] The bulk heterojunction PV cells fabricated in this study had a device structure of ITO/ PEDOT-PSS/ PCDTBT: C60 derivatives 1 or 5 / LiF (1 nm)/ Al (120 $\mathrm{nm})$ with a PV active area of $1 \mathrm{~cm}^{2}$. The active layer was spin-cast from dichlorobenzene solution using a slow solvent evaporation process. The weight ratio of PCDTBT to $\mathrm{C} 60$ derivatives varies from $1: 1$ to $1: 3$. Because of the poor solubility of the C60 derivative 5 in dichlorobenzene, it was difficult to get a smooth and uniform film, and the resulting device had a very low PV response. For PCDTBT/C60F 1 based solar cells, the best performance was achieved at a 1:2 weight ratio, and the power conversion efficiency reached $2.3 \%$ with a Voc of 0.90 $\mathrm{V}$ and a Jsc of $6.4 \mathrm{~mA} / \mathrm{cm}^{2}$. This performance was lower than PC61BM based devices (4.5\% efficiency). Detailed analysis of the dark I-V curves of the C60 derivative $\mathbf{1}$ based devices revealed that the device series resistance is too high (39 $\Omega \mathrm{cm}^{2}$ ), leading to a low fill factor $(0.40)$. We found that the field-effect electron mobility of the C60 derivative 1 was two orders of magnitude lower than that of PC61BM.
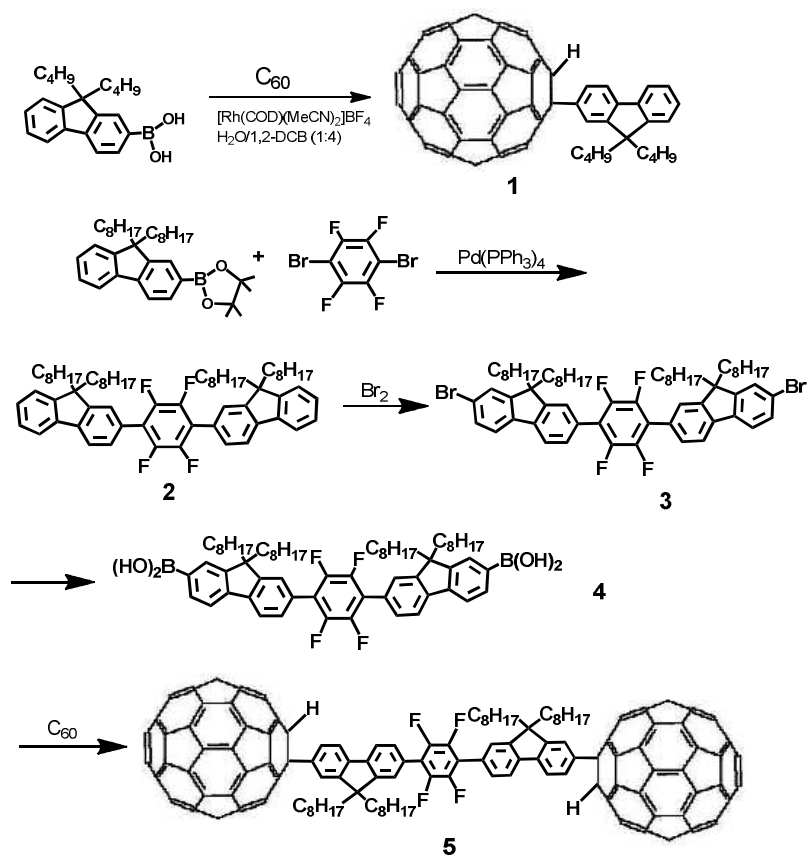

Scheme 1. Synthetic Route to oligofluorene modified C60 derivatives

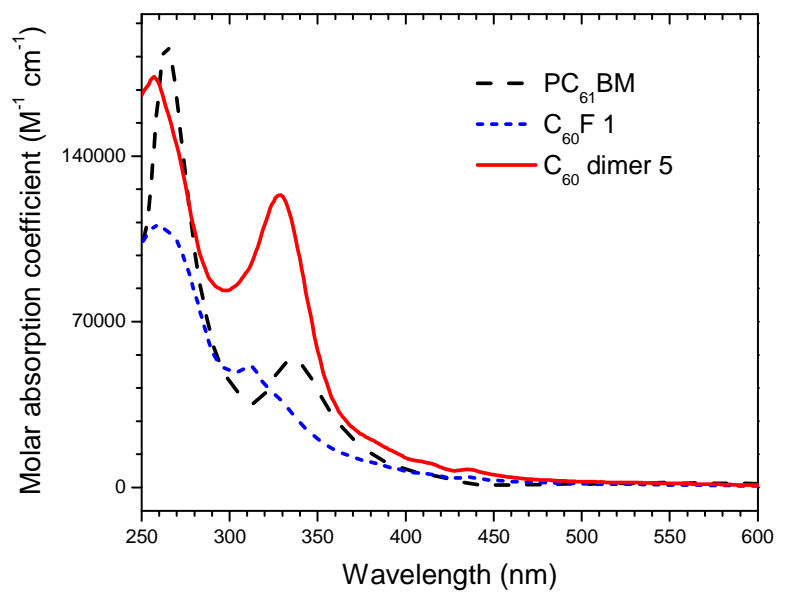

Figure. $1 \mathrm{UV}$-vis absorption spectra in chloroform of C60 derivatives 1, 5 and PC61BM.

\section{B. 5-alkoxyindene-fullerene monoadduct Margins}

The cycloaddition reaction between indene and C60 fullerene in refluxing 1,2-dichlorobenzene has been reported in the literature. Recently, several groups independently reported that at higher reaction temperatures and longer reaction time, two indene molecules can be attached to one C60 cage. Because the 
number of the $\pi$ electrons in this indene-fullerene bisadduct (IC60BA) is reduced to 56, ICBA has a higher LUMO energy level than widely used PC61BM. As a result, the BHJ solar cell based on the Poly(3hexylthiophene) (P3HT)/IC60BA blend offered a large open-circuit voltage ( $\mathrm{Voc}$ ) of $0.84 \mathrm{~V}$, compared with 0.58 $\mathrm{V}$ for the P3HT/PC61BM system. In addition, the overall power conversion efficiency was increased from $3.9 \%$ to 5.4\%. [7-8] Although the ICBA/P3HT blend works well, ICBA does not match with the low-bandgap p-type polymers that have low-lying LUMO energy levels because the energy offset between the LUMO energy levels of the polymer and ICBA are too small to provide enough driving force for efficient charge separation in bulk heterojunction solar cells. As a result, the photocurrent is pretty low. For example, the efficiency of PCDTBT/IC60BA based devices was less than $1 \%$. As to the indene-fullerene monoadduct (ICMA), its LUMO energy level is suitable for most low-bandgap p-type polymers. However, its solubility is not high enough for solution processing. Therefore, there remains a need for new fullerene derivatives having improved solubility and good electron mobility for use in organic solar cells.

In this work, we synthesized novel soluble fullerene derivatives (including both C60 and C70) by a controlled cycloaddition reaction between fullerenes and indenebased compounds containing electron donating groups, as shown in scheme 2. We demonstrated that with the electron-donating substituents, the solubility of the resulting fullerene derivatives was greatly improved. Moreover, these polar functional groups also facilitated the product purification process. The fullerene derivatives were prepared in 1,2,4-trichlorobenzene at $185{ }^{\circ} \mathrm{C}$ for $5-8$ h. The 5-alkoxyindene-fullerene monoadducts can be easily separated from unreacted fullerene and other byproducts by silica gel chromatography using the mixture of toluene/hexanes as the eluent due to their different polarity. In contrast, it is difficult to separate unsubstituted indene-fullerene monoadduct from unreacted fullerene and other byproducts because of their similar polarity. The purity of the purified fullerene derivatives was tested on HPLC equipped with a Cosmosil Buckyprep column (10.0 $\mathrm{mm} \times 250 \mathrm{~mm}$, Nacalai USA), and found to be above 99.5 $\%$.<smiles>COc1ccc2c(c1)C(=O)CC2</smiles>

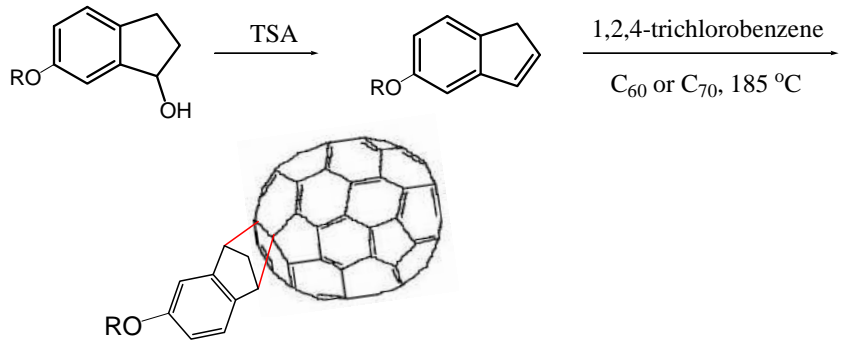

Scheme 2. Design and synthesis of 5-alkoxyindene-fullerene monoadduct.
The photovoltaic (PV) performance of 5-methoxyindeneC70 monoadduct (MOIC70MA) and 5-butoxyindeneC70 monoadduct (BOIC70MA) was tested in BHJ solar cells using PCDTBT as a p-type material. For comparison purpose, unsubstituted indene-C70 monoadduct (IC70MA) and widely used PC71BM were also blended with PCDTBT under the same condition. Figures 2 and 3 show the current-voltage characteristics and external quantum efficiency (EQE) curves of the fabricated solar cells, respectively. Detailed analysis of the dark I-V curves revealed that the device series resistance decreased from $10 \Omega \mathrm{cm}^{2}$ for the PC71BM based device to 4 and $5 \Omega \mathrm{cm}^{2}$ for the MOIC70MA and BOIC70MA based devices, respectively. As a result, the device fill factor increased from 0.60 to 0.64 for BOIC70MA and even surprising 0.69 for MOIC70MA, and the overall EQE-calibrated power conversion efficiency was enhanced from $5.6 \%$ to $6.2 \%$. For the IC70MA based device, an ideal interpenetrating electron donor/acceptor network can't be achieved because of its poor solubility. As a result, the device fill factor (0.5) was low, the photocurrent only reached $8.8 \mathrm{~mA} / \mathrm{cm}^{2}$, and the overall power conversion efficiency was only $4.0 \%$, which was significantly lower than the MOIC70MA based device.

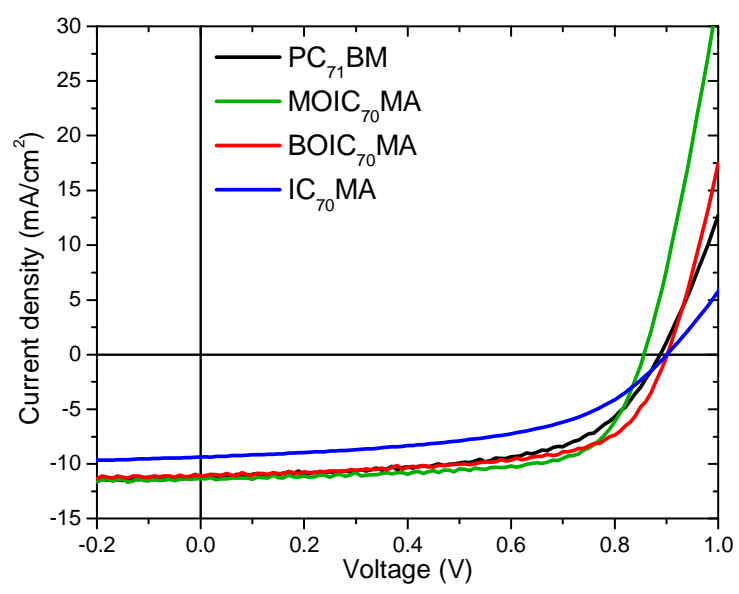

Figure 2. Photocurrent-voltage characteristics of the $\mathrm{C} 70$ derivatives:PCDTBT based solar cells.

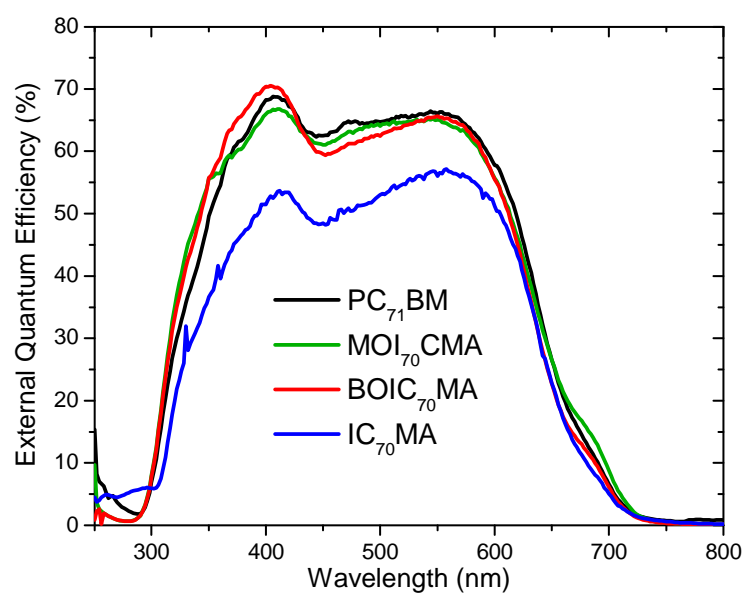

Figure 3. External quantum efficiency of the C70 derivatives:PCDTBT based solar cells. 


\section{Conclusion}

In summary, we have demonstrated two facile approaches for the chemical modification of fullerenes via either Rhcatalyzed arylation with organoboronic acids or cycloaddition with 5-alkoxyindene. UV-vis absorption and CV measurements showed that the substituents have little impact on the energy levels of the resultant fullerene derivatives. However, we found that the substituents have huge impacts on other physical properties, such as solubility and crystallinity. Since the control of film morphology is critically important for applications in organic electronic devices, the substituents of the fullerene derivatives can be tailored to achieve desired nanostructured morphology when blended with semiconducting polymers.

\section{Acknowledgement}

The authors would like to thank Judy Kung and Ludmila Scoles at EME-NRC and Raluca Movileanu at AEP-NRC for their technical support. The financial support from the Printable Electronics program of National Research Council of Canada is greatly appreciated.

\section{References}

[1] Y. Liu, J. Zhao, Z. Li, C. Mu, W. Ma, H. Hu, K. Jiang, H. Lin, H. Ade, H. Yan, Nature Commun. 2015, 5, 5293.

[2] H. Cha, J. Wu, A. Wadsworth, J. Nagitta, S. Limbu, S. Pont, Z. Li, J. Searle, M. Wyatt, D. Baran, J. Kim, I. McCulloch, J. Durrant, Adv. Mater. 2017, 29, 1701156.

[3] K. M. Kadish and R. S. Ruoff, Eds. Fullerenes: Chemistry, Physics, and Technology; Wiley: New York, 2000.

4] F. Diederich and R. Kessinger, Acc. Chem. Res., 1999, 32, 537-545.

[5] M. Nambo, R. Noyori, and K. Itami, J. Am. Chem. Soc., 2007, 129, 8080-8081.

[6] S. H. Park, A. Roy, S. Beaupré, S. Cho, N. Coates, J. S. Moon, D. Moses, M. Leclerc, K. Lee and A. J. Heeger, Nature Photonics, 2009, 3, 297-303.

[7] He, Y.; Chen, H.; Li, Y. J. Am. Chem. Soc. 2010, 132, 1377-1382.

[8] Laird, D. W.; Richter, H.; Vejins, V.; Scott, L.; Lada, T. A. US patent, US 2009/0176994 A1. 